\title{
The Measurement Problem and two Dogmas about Quantum Mechanics
}

Laura Felline

\begin{abstract}
According to a nowadays widely discussed analysis by Itamar Pitowsky, the theoretical problems of QT are originated from two 'dogmas': the first forbidding the use of the notion of measurement in the fundamental axioms of the theory; the second imposing an interpretation of the quantum state as representing a system's objectively possessed properties and evolution. In this paper I argue that, contrarily to Pitowsky analysis, depriving the quantum state of its ontological commitment is not sufficient to solve the conceptual issues that affect the foundations of QT.

In order to test Pitowsky's analysis I make use of an argument elaborated by Amit Hagar and Meir Hemmo, showing how some probabilistic interpretations of QT fail at dictating coherent predictions in Wigner's Friend situations. More specifically, I evaluate three different probabilistic approaches: qBism, as a representative of the epistemic subjective interpretation of the quantum state; Jeff Bub's information-theoretic interpretation of QT, as an example of the ontic approach to the quantum state; Itamar Pitowsky's probabilistic interpretation, as an epistemic but objective interpretation. I argue that qBism succeeds in providing a formal solution to the problem that does not lead to a selfcontradictory picture, although the resulting interpretation leads to an interpretation where the real subject matter of QT clashes alarmingly with scientific practice. The other two approaches, instead, strictly fail in Wigner's Friend scenarios, showing in such a way that they don't provide a genuine solution to the problem.
\end{abstract}

\section{Introduction}

There is a time-honoured approach to the foundations of Quantum Theory (QT), according to which the oddities of this theory originate from the improper interpretation of its subject study as concerning properties and behaviour of material objects, being them corpuscles or waves, represented by the quantum state. This interpretation is often held responsible, for instance, for the difficulties in explaining quantum correlations, or the measurement problem.

The argument behind this association is quite intuitive. Let's take the problem of (apparently) non-local quantum correlations, and the illustrative case of an EPR-Bohm entangled pair of particles A and B, which are sent apart. The EPR-Bohm state does not associate a determinate property to either particle, however, when a measurement on $\mathrm{A}$ is performed, the entangled state of $\mathrm{A}+\mathrm{B}$ instantaneously collapses, associating now a specific value to the measured observable, not only in the system A, but in the system B as well. As a result, due to A's and B's perfect anticorrelation, knowing the result of a measurement on A automatically also informs us about the state of B. 
Now, if the quantum state is interpreted realistically, as encoding the properties possessed by a system, and its evolution, then the collapse represents a physical change both in A's and in B's properties. But B was never disturbed by the observer's interaction with A, so an explanation is in order for B's behaviour. At the origin of the difficulty of the sought-after explanation is the tension between the natural assumption that the measurement over A has in some way caused the change in B's state, and the fact that causal processes can't be instantaneous.

This difficulty seems to disappear once we give up the assumption that the quantum states, like classical states, represent properties and evolution of physical systems. If the quantum state is just an economical codification of a probability distribution over measurement results, then the collapse of the wave function does not represent an actual physical process, and B's state change does not have to be explained physically.

So, according to this argument, the problem of non-locality (and, as we are about to see, the measurement problem with it) is explained away once one rejects what I will call from now on a representational function of the quantum state. ${ }^{1}$

But this conclusion would be too quick. For instance, in the case of non-locality, a genuine solution to the problem must necessarily provide an explanation of why, given that there is no physical process connecting the occurrence of A's and B's measurement results, said results are always perfectly anti-correlated. But this is not an easy task. As a matter of fact, when it comes to provide an alternative explanation to quantum conundrums, non-representational approaches plod along as well.

Because of these persisting difficulties, mainstream physics settles for the adoption of a pragmatic but inconsistent attitude towards the quantum state (see Wallace 2016), to be interpreted as representing a system or as a codification of probabilities of measurement results, depending on what works in that situation.

This, for instance, is physicist David Mermin, about the two-slit experiment:

"We know what goes through the two slits: the wave-function goes through, and then subsequently an electron condenses out of its nebulosity onto the screen behind. Nothing to it. Is the wave-function itself something real? Of course, because it's sensitive to the presence of both slits. Well then, what about radioactive decay: does every unstable nucleus have a bit of wave-function slowly oozing out of it? No, of course not, we're not supposed to take the wave-function that literally; it just encapsulates what we know about the alpha particle. So then it's what we know about the electron that goes through the two slits? Enough of this idle talk: back to serious things!" (Mermin, 1990 p. 187, cited in Maudlin, 1997, p. 146)

\footnotetext{
${ }^{1}$ See later in this section ad especially note 2 for some clarifications about the terminology.
} 
This being said, in the last decades non-representational approaches to QT have experienced new life thanks to the evolutions of Information Theory. The hope is that the latter might provide a formal background powerful enough to allow a genuine solution to the theoretical problems of QT, without necessarily collapsing into instrumentalism. Accordingly, QT is not about particles or waves and their behaviour, but is rather about information.

A terminological clarification is in order at this point. The approaches that deny that QT is about properties and behaviour of physical entities are often labelled anti-realist approaches to QT (see e.g. Myrvold 2018, §4.2); however, this characterization might be confusing at times, since not every such an account is anti-realist. In fact, the majority of them openly rejects anti-realism (and this is definitely true of the three approaches examined in this paper): QT is not a theory about the elementary constituents of matter, but it is still realist with respect to its subject matter.

In this paper, I adopt Jeff Bub's terminology and label the two competing views probabilistic and representational approaches, where the former interprets QT as a theory of probability (and where we can further distinguish between an ontic and an epistemic interpretation of the quantum state), while the latter interpret QT as a theory about the elementary constituents of matter, their properties and their dynamical behaviour. ${ }^{2}$

As a more general point, I argue that what was already shown for more traditional probabilistic approaches, is valid also for the new information-based formulations: contrarily to the simplistic attitude often adopted even in recent illustrations of the subject, depriving the quantum state of its ontological commitment is not sufficient to solve the conceptual issues that affect the foundations of QT.

In arguing for this general point, I focus on the measurement problem, typically taken to be explained away quite easily in non-representational accounts. As in the above illustrated example of non-locality, and contrarily to what often claimed by the literature, also in the case of the measurement problem, the majority of non-representational accounts fail to provide a genuine solution.

One of the most systematic expositions of the non-representational view and of its relationship with the measurement problem was put forward few years ago by Itamar Pitowsky, in a series of paper (Pitowsky 2003, 2004, 2006; Bub and Pitowsky 2010) where he puts forward his own Bayesian theory of quantum probability.

\footnotetext{
${ }^{2}$ A further clarification: Bub takes this terminology from Wallace (2016) where, however, probabilistic interpretations are characterized as those according to which observables always have a determinate values, while the quantum state is merely "an economical way of coding a probability distribution over those observables" (p.20). I think this characterization is incorrect of the three approaches here analysed, since they do not take observables as determinate all the time, therefore I don't refer to Wallace terminology.
} 
According to his analysis, the theoretical problems of QT are originated from two 'dogmas': the first forbidding the use of the notion of measurement in the fundamental axioms of the theory; the second imposing an interpretation of the quantum state as representing a system's objectively possessed properties and evolution.

In this paper I illustrate and criticise Pitowsky's analysis, taking the rejection of the two dogmas as an accurate articulation of the fundamental stances of the probabilistic approach to the measurement problem. In order to assess such stances, I will test them through the use of an argument elaborated by Amit Hagar and Meir Hemmo (2006), showing how some probabilistic interpretations of QT fail at dictating coherent predictions in Wigner's Friend situations.

More specifically, I evaluate three different probabilistic approaches: qBism (but more specifically, the version articulated by Christopher Fuchs, e.g. Fuchs 2010, Fuchs et al. 2014) as a representative of the epistemic subjective interpretation of the quantum state; Bub's informationtheoretic interpretation of QT (Bub 2016, 2018) as an example of the ontic approach to the quantum state; Pitowsky's probabilistic interpretation (Pitowsky 2003, 2004, 2006, 2010), as an epistemic but objective interpretation. I argue that qBism succeeds in providing a formal solution to the problem that does not lead to a self-contradictory picture, although the resulting interpretation would bring the real subject matter of QT to clash alarmingly with scientific practice. The other two approaches, instead, strictly fail when confronted to scenarios where the measurement problem is relevant, showing in such a way that they don't provide a genuine solution to the problem.

\section{Two dogmas and two problems}

The idea that deflating the quantum state's ontological import is sufficient to explain away the measurement problem is a leitmotif in the literature about information-based accounts of QT, still shared by the vast majority of the advocates of the probabilistic view:

"The existence of two laws for the evolution of the state vector becomes problematical only if it is believed that the state vector is an objective property of the system. If, however, the state of a system is defined as a list of [experimental] propositions together with their [probabilities of occurrence], it is not surprising that after a measurement the state must be changed to be in accord with [any] new information.” (Hartle 1968, as quoted in Fuchs 2010)

Itamar Pitowsky, also together with Jeff Bub (Pitowsky 2006, Bub and Pitowsky 2010), has identified two distinct issues behind the measurement problem. The first one, that he calls the small measurement problem, is the question "why is it hard to observe macroscopic entanglement, and what are the conditions in which it might be possible?" $(2006,28)$ The second issue, the big measurement problem, is the problem of accounting for the determinateness of our experiences: 
"The 'big' measurement problem is the problem of explaining how measurements can have definite outcomes, given the unitary dynamics of the theory: it is the problem of explaining how individual measurement outcomes come about dynamically." (Bub and Pitowsky, 2010, p.5)

In this paper we are exclusively concerned with the big problem; therefore, from now on, we will refer to the latter more simply as the measurement problem.

The second most notable contribution that Pitowsky has given to the analysis of the measurement problem concerns the identification of two assumptions, labelled as 'dogmas', in the interpretation of QT, that, according to the philosopher, are at the origin of the issues in the interpretation of QT.

“The first dogma is Bell's assertion (defended in [(Bell 1987)]) that measurement should never be introduced as a primitive process in a fundamental mechanical theory like classical or quantum mechanics, but should always be open to a complete analysis, in principle, of how the individual outcomes come about dynamically. The second dogma is the view that the quantum state has an ontological significance analogous to the ontological significance of the classical state as the 'truthmaker' for propositions about the occurrence and non-occurrence of events, i.e., that the quantum state is a representation of physical reality." (Pitowksy 2010, 5)

A couple of observations are in order here. First of all, the two claims: "the quantum state has an ontological significance analogous to the ontological significance of the classical state as the 'truthmaker' for propositions about the occurrence and non-occurrence of events" and "the quantum state is a representation of physical reality", are not equivalent, although they are here put forward as such. More specifically, one can deny that the quantum state represents 'traditionally' (Bub 2018) in the sense that it does not represent a system's properties, and therefore acting as a truthmaker for propositions about the occurrence and non-occurrence of events, without denying that the quantum state represents physical reality. In fact, that's the case for Bub's informationtheoretic interpretation which, as I will argue below, denies that the quantum state represents a system's properties and dynamics, and yet interprets the quantum state ontically, as representing physical reality. In the following, therefore, we take the second dogma as the view that the quantum state does not represent traditionally, while the issue whether the quantum state represents physical reality or a mental state remains open.

Secondly, although probabilistic interpretations reject both dogmas, it is useful to keep in mind that, at least at a first sight, the two are logically distinct, and it still remains to be seen whether they need to come in package. The rejection of the first dogma leads to a black-box interpretation, whose traditional versions put forward an interpretation of the quantum state as representing the properties of physical systems (e.g. Bohr's interpretation). It might be argued that the more traditional black- 
box theories have proven inconsistent, and that once one rejects the first dogma, the explanation away of the measurement problem requires the rejection of the second. This is the, for instance, the core of Pitowsky's and Bub's view, according to which not only the second dogma runs 'very quickly' into the measurement problem (Pitowsky 2006, p.3), but the rejection of the latter is sufficient to solve the measurement problem in black-box theories.

In support of this last claim, over which the critical analysis of this paper is pivoting, Pitowsky outlines a straightforward argument. First of all, the measurement problem consists in the tension between the fact that measurements always yield determinate values, and the fact that they are also performed on systems whose states do not associate a determinate value to the measured observable. If this is so, for the achievement of a solution it would be sufficient to reject one of the horns of the dilemma:

"“"The BIG problem concerns those who believe that the quantum state is a real physical state which obeys Schrodinger's equation in all circumstances. In this picture a physical state in which my desk is in a superposition of being in Chicago and in Jerusalem is a real possibility; and similarly, a superposed alive-dead cat. [...]

In our scheme quantum states are just assignments of probabilities to possible events, that is, possible measurement outcomes. This means that the updating of the probabilities during a measurement follows the Von Neumann-Luders projection postulate and not Schrodinger's dynamics. [...] So the BIG measurement problem does not arise.” (Pitowsky 2006, 26-27)

\section{Incompatible predictions in black-box approaches}

In this section I am going to introduce a somewhat neglected argument put forward in (Hagar 2003) and in (Hagar and Hemmo 2006), which introduces a scenario against which, according to $\mathrm{HH}$, black-box interpretations of QT fail.

Let's take an experiment with two observers, Wigner and Friend, where the latter is inside a lab, isolated from Wigner and the rest of the environment. Before Friend enters the lab, she and Wigner agreed upon the following protocol: in the first part of the experiment Friend performs a z-spin measurement on a half-spin particle $\mathrm{P}$ in the state:

$$
\mid \text { Psi } 0>_{\mathrm{P}}=\left(1 / \sqrt{ } 2\left|+_{\mathrm{z}}>_{\mathrm{P}}+1 / \sqrt{ } 2\right| \mathrm{-}_{\mathrm{z}}>_{\mathrm{P}}\right)
$$

Therefore, the system composed of Friend and the particle $(\mathrm{F}+\mathrm{P})$ is in the state:

$$
\mid \text { Psi } 0>_{\mathrm{P}+\mathrm{F}}=\left(1 / \sqrt{ } 2 \alpha\left|+_{\mathrm{Z}}>_{\mathrm{P}}+1 / \sqrt{ } 2 \beta\right|-_{\mathrm{Z}}>_{\mathrm{P}}\right) \mid \text { Psi } 0>_{\mathrm{F}}
$$

Since they agreed on this first part of the protocol, Wigner knows that Friend is going to perform the measurement; however, because the lab is isolated from Wigner and the rest of the environment, he does not know the result of her measurement. 
Now, consider a second measurement, of an observable $\mathrm{O}$ of the system $\mathrm{F}+\mathrm{P}$, with eigenstate

$$
\left|\mathrm{Psi}>_{\mathrm{P}+\mathrm{F}}=1 / \sqrt{ } 2\right|+\mathrm{Z}>_{\mathrm{P}}\left|+\mathrm{Z}>_{\mathrm{F}}+1 / \sqrt{ } 2\right|-\mathrm{Z}>_{\mathrm{P}} \mid-\mathrm{Z}>_{\mathrm{F}}
$$

whose eigenvalue is, say, YES.

HH's question is: according to a black-box approach, what predictions are dictated by QT for the results of this measurement?

On the one hand, $\mathrm{F}+\mathrm{P}$ is an isolated system; therefore, its evolution should follow Schrodinger equation. If so, the state at the end of Friend's measurement should be

$$
\mid \text { Psi } 1>_{\mathrm{P}+\mathrm{F}}=1 / \sqrt{2}\left|+\mathrm{Z}>_{\mathrm{P}}\right|+\mathrm{Z}>_{\mathrm{F}}+1 / \sqrt{ } 2\left|-\mathrm{Z}>_{\mathrm{P}}\right|-\mathrm{Z}>_{\mathrm{F}}
$$

which is an eigenstate of $\mathrm{O}$, with eigenvalue YES. From this perspective, therefore, QT dictates that the measurement of $\mathrm{O}$ will yield YES with probability 1.

On the other hand, Friend's interaction with $\mathrm{P}$ is a measurement interaction, which, according to the black-box approach, requires the application of Luders' rule. It follows therefore that F+P's state should collapse into either one of two pure states

$$
\mid \text { Psi } 1>=\left|+\mathrm{Z}>_{\mathrm{P}}\right|+\mathrm{Z}>_{\mathrm{F}}
$$

or

$$
\mid \text { Psi } 1>=\left|-\mathrm{z}>_{\mathrm{P}}\right|-\mathrm{z}>_{\mathrm{F}}
$$

both associating a probability $1 / 2$ to the result YES in an O-measurement. According to this line of thought, therefore, QT dictates that the probabilities for a YES result over the measurement of O is $1 / 2$.

To make things more complicated, each option adopts a specific stance about the evolution of the quantum state in a measurement process, ruling therefore out a black-box approach. If we take into consideration that the lab is an isolated system, and therefore apply Schrodinger equation to the first part of the experiment, this would correspond to a no-collapse approach, since Friend is failing to update her quantum state after performing a measurement. If, on the opposite, we apply Luders rule, then we are indeed applying a collapse view of quantum measurement. ${ }^{3}$

According to Hagar and Hemmo $(\mathrm{HH})$, this shows that any solution to this kind of Wigner's friend scenario (and therefore, a fortiori, of the measurement problem) implies an analysis of the processes behind measurement interaction. Since, they add, every black-box interpretation of QT is equally affected by this argument, they conclude that black-box approaches to quantum theory are unable to provide a coherent picture of the predictions of QT.

\footnotetext{
${ }^{3}$ It might be useful to anticipate that one way to block the conclusion of HH's argument is to bring into question the possibility to verify the contradictory predictions. In this paper I do not face this kind of objections, but the interested reader should refer to a series of recent arguments based on more sophisticated Wigner's friend kind of thought experiments designed to overcome this kind of objections, e.g. (Frauchiger and Brenner 2018) or (Baumann and Brukner 2019).
} 
In the rest of the paper, the application of this argument to three different theoretical accounts will be used to explore the relationship between the two dogmas and the measurement problem.

\section{Qbism}

We have already said that $\mathrm{HH}$ chose qBism as a case study to formulate their argument, while claiming that the same result applies to any black-box approach to QT. The succeeding debate around this specific approach, however, has shed some light about how different black-box approaches lead to different results when faced to Wigner's Friend scenarios. In order to formulate my criticism of qBism, in the following I will build on such a debate. The aim will be to isolate the exact feature of qBism that allows it to formally escape HH's argument, and show how this same feature leads to a philosophically problematic account of QT.

According to qBism, the quantum state represents the rational subjective belief of an observer, assembled from the personal experience gathered by each individual. The first direct consequence of this interpretation is that quantum probabilities are not a measure of anything in reality, but only of the degree of belief of an observer over future experience. Secondly, different observers might consistently attribute different quantum states to the same system. In order to solve the dilemma in the Wigner's Friend scenario, therefore, one needs to take into consideration each observer's individual perspective: given that she has gathered new data, QT prescribes Friend to update P's quantum state (and F+P's state, clearly). From her perspective, then, the prediction of the result in an O-measurement in the second stage of the experiment will be YES with probability $1 / 2$. On the other hand, Wigner has registered no new data, therefore QT prescribes him to maintain the entangled state (4) for F+P. From his perspective, therefore, the O-measurement will yield YES with probability 1 .

The surprising consequence is that, according to qBism, different observers can coherently hold incompatible predictions about the world. The subjectivism embraced by qBism turns QT into "a "single-user theory": probability assignments express the beliefs of the agent who makes them, and refer to that same agent's expectations for her subsequent experiences. The term "single-user" does not, however, mean that different users cannot each assign their own coherent probabilities." (Fuchs et al. 2014, p. 2)

According to Amit Hagar (2007) this strategy can't work: the (repeated) measurement of O will yield a series of results with a certain frequency that will (hopefully!) confirm one of the predictions and falsify the other. If this is so, at the end of the day one of the two alternatives (collapse or nocollapse) will be right, while the other will be wrong.

To Hagar, Timpson (2013) replies that this kind of objections are ineffective against Qbism: 
"[n]o objection can be successful which takes the form: "in such and such a situation, the quantum Bayesian position will give rise to, or will allow as a possibility, a state assignment which can be shown not to fit the facts', simply because the position denies that the requisite kind of relations between physical facts and probability assignments hold" (Timpson 2013, p. 209).

Here is my attempt to unpack Timpson's position: in the experiment here illustrated, the consequence of the subjective interpretation of probabilities is that Wigner's beliefs about the quantum state of $\mathrm{F}+\mathrm{P}$ are partially determined by a previous subjective assignment, therefore the 'probability 1' of the result YES is also merely subjective, rather than an objective certainty. Let's say for instance that Wigner's experiment yields a different result from YES. According to an ontic view of the quantum state, probabilistic predictions are determined by an objective reality that is grasped by the theory. Wrong predictions, therefore, show that there is something wrong in Wigner's representation of reality: this is one of the pillars of empirical sciences reasoning. However, in qBism, the relation that holds between data and quantum state representation is not of this kind; probabilities do not represent anything in the world, which means that Wigner's quantum state representation can't be wrong about the world. As a consequence, dealing with 'surprising' experiences does not have to be cause of sweat for the qBist, who's reaction is simply to apply the same rules for updating expectations (s)he applies every time (s)he must associate (but not discover) previously unknown chances to an event.

However, one can still counter that, after a sufficiently large repetition of the experiment, the falsification (on either side) of one's predictions should lead a rational agent to abandon the unsuccessful rules she has been following for the formation of expectations (i.e. QT!). ${ }^{4}$ But this means, as $\mathrm{HH}$ argued, that QT is, as it is, incomplete.

Another counterargument often used to block HH's conclusion is to hinge on the fact that there is no way for Wigner and Friend to compare their experience and predictions: "Results objectively available for anyone's inspection? This is the whole issue with "Wigner's friend" in the first place. If both agents could just "look" at the counter simultaneously with negligible effect in principle, we would not be having this discussion." (Fuchs and Stacey 2019, 9)

The argument here is that in the second measurement, P's z-spin and Friend's record of it will be erased, so that any ground for Friend's $1 / 2$ predictions would fail. However, that this is not really the core of the issue has been already argued extensively, ${ }^{5}$ and recently explicitly illustrated by more sophisticated versions of the Wigner's friend thought experiment showing that the same kind of

\footnotetext{
${ }^{4}$ I am very grateful to Meir Hemmo and an anonymous referee for pushing me to provide a more thorough evaluation of Timpson's position.

${ }^{5} \mathrm{By} \mathrm{HH}$ themselves first, but see also, just to cite one, (Bacciagaluppi, 2013) for an insightful take on the problematical character of the qBist solution.
} 
contradicting predictions can be reproduced even in scenarios where the necessary information is available to all the observers, while the lab is maintained in isolation (Frauchiger and Renner, 2018).

The core of the qBist solution to this problem lies instead in "some kind of solipsism or radical relativism, in which we care only about single individuals' credence's, and not about whether and how they ought to mesh" (Bacciagaluppi 2013,6)

Now, it is difficult to formulate an argument with solid grounds in favour of an interpretation of QT, if your starting point is solipsism. However, it might be argued that the situation is not as bad as it might seem. Timpson (2013, 9.3, but see also Healey 2017, §2), for instance, denies that qBism implies solipsism, and claims instead that the former avoids the paradoxical or anyway pernicious consequences of the latter as a general epistemological thesis. In other words, although, according to qBism, QT fails to include the perspective of more than one observer, it does not follow that according to Qbism there is only one sentient being in the world, let alone that the rest of empirical science is affected by these limitations. This same defence strategy is used by Timpson against the charge of instrumentalism: it is true that, according to Qbism, QT is neither true or false of the world, and a mere instrument for bookkeeping of probabilities. However, this conclusion follows, in the case of Qbism, from specific considerations about the structure of QT and only applies to said theory. Because of these considerations, qBism does not imply the majority of the controversial features of instrumentalism as a general position about science.

The upshot of this rebuttal to criticisms is that: QT might forbid inferences about the experiences of other observers as solipsism does, and it might be as void of knowledge about the world, as instrumentalism dictates - but this is OK, because does qBism not lead to the possibly paradoxical consequences of such thesis.

I am not going here to challenge Timpson's argument. Here, I take for granted that this defence provides qBism with a way-out from the conclusions of $\mathrm{HH}$ argument. This being said, given the place where we ended up, it is legitimate to scrutinize the consequences of this unique status of QT with respect to the rest of empirical science - uniqueness that, we just concluded, lies at the core of the qBist defence.

I am going to argue that this achievement is a pyrrhic victory, as it still fails to provide an acceptable picture of the scientific enterprise in the field of QT.

If qBism succeeds in the context of Wigner's Friend scenarios, but also in explaining away other apparent oddities of the quantum world, it characterizes QT not as a physical theory, but as a Bayesian theory for the formation of expectations. As such, it can coherently ignore the constraints imposed to an objective description of the world, when they are in the way of coherence. While a 
theory about the world must submit to the typical epistemic and methodological standards of empirical sciences, Bayesian epistemology is subordinate to very different standards, for the simple reason that different subject studies require different methodologies. The violation of the requirement of intersubjectivity in the qBist explanation away of non-locality (see Fuchs et al. 2014) and in the account of the Wigner's Friend thought experiment.

In other words, Qbism plays a different game, with different rules, with respect to physics and, more in general, with respect to natural sciences. And it escapes strict failure when confronted against HH's argument (as far as self-consistency is the only requirement to avoid strict failure, but see later) only as far as QT is not interpreted as natural science.

If this is so, a coherent and truthful endorsement of Qbism implies also the renunciation to constraints whose great value is of guidance to scientific change like empirical adequacy, physical salience, or intersubjectivity because, in a quantum Bayesian theory about rational belief, such criteria fail to make sense. In fact, the renunciation to such criteria is the key for the Qbist explanation away of the oddities of QT.

And yet, it can't be ignored that giving up these criteria means throwing away a huge piece of scientific practice - a hard bullet to bite for working physicists using QT as an empirical science every day.

One might reply that this is a made-up problem: scientific practice does not, nor should, change because of philosophical debates, so there is nothing to be worried about. However, the unproblematic acknowledgment of such a chasm between scientific practice and the real content of scientific theories, is an even harder bullet to bite (if not straightforward failure) for philosophers. ${ }^{6}$

This is what one gets when submitting to a Qbist view of QT. As we will see in the next sections, those that indeed think that this is too high price to pay for internal consistency, but still want to deny the representational role of QT, will have to do so while maintaining in some way the empirical import of the quantum state, denied by Qbism.

\footnotetext{
${ }^{6}$ In his analysis of the virtues and problems of Qbism, Chris Timpson formulates a similar challenge to Qbism, but focusing on explanation:

"It seems that we do have very many extensive and detailed explanations deriving from quantum mechanics, yet if the quantum Bayesian view were correct, it is unclear how we would do so." (Timpson 2013, 226)

Timpson's criticism is that Qbism suffers of an explanatory deficit because it can't explain why QT explains; however, I think that the Qbist lack of a realist explanation for the explanatory power of QT is hardly an unsurmountable problem.

First of all, the Qbist can reply to Timpson's challenge that there is no reason why a theory that is neither true nor false can't do what false theories have done for centuries: being explanatory. If then one insists (wrongfully, I think) that only at least approximate truth have explanatory power, then the Qbist can simply deny a genuine explanatory power for QT: after all, the topic of explanation in QT is a time-honoured headache in philosophy of science, and the philosophers that deny the explanatory power of QT are not few. An objection hinging on the explanatory power of QT can go just as far.
} 


\section{Bub's information-theoretic interpretation of QT}

According to Jeff Bub, QT is a non-representational, probabilistic theory. Yet, while rejecting a traditional representational interpretation of the quantum state, this information-theoretic approach interprets the quantum state ontically, as a physical state, a complete description of a quantum system (Bub 2016, 222). More specifically, QT is a theory about information 'in the physical sense': a structure of correlations between intrinsically random events, which is different from the classical structure of correlations measured by Shannon information. Moreover, according to Bub, information is a new kind of physical primitive, whose structure "imposes objective pre-dynamic probabilistic constraints on correlations between events, analogous to the way in which Minkowski space-time imposes kinematic constraints on events." (Bub 2018, p.5)

Bub adopts and contributes to Pitowsky's analysis about the two dogmas (Bub and Pitowsky 2010; Bub 2016, § 10) and in fact he also puts them at the origin of the issues gravitating around the measurement problem. However, the application of HH's argument to Bub's information-theoretic interpretation shows that the rejection of the two dogmas is not sufficient to solve the measurement problem.

In HH's thought experiment, in fact, QT provides contrasting instructions for the prediction of measurement results. The qBist way out to inconsistency is relativizing the quantum state to single observers. In Bub's interpretation, on the other hand, the quantum state represents an objective probabilistic structure that constrains the system's behaviour. This means that the probabilities codified by QT are objective, and therefore that different observers must associate the same measurement with the same predictions. In Bub's account, QT is not a single-user theory: through its use, Wigner and Friend must be able to come to agreement.

Let's wrap up the results achieved so far. Contrarily to HH's conclusions, their Wigner's Friend thought experiment does not rule out black-box interpretations of QT; however, Qbism escapes failure by embracing an extremely subjective view of the quantum state that, although dragging QT out of the realm of empirical sciences, allows to consistently disattend constraints imposed to such sciences. Bub, on the other hand, puts forward an information-theoretic account of QT which has the merit of maintaining the empirical import not only of Hilbert space, but also of the quantum state. Regaining the empirical import of the theory, however, means losing the possibility to exploit the qBist solution to HH's test, because, as any other empirical theory, it must be empirically adequate and provide an intersubjective description of the non-perspectival features of our experiences.

In the next section I will discuss Pitowsky's information-theoretic interpretation, a kind of compromise in between the extreme subjectivism of Qbism, and Bub's ontic approach. 
It has to be said that Bub has recently addressed the issue of Wigner's Friend scenarios in the information-theoretic interpretation. However, since this new contribution have several points of intersection with Pitowsky's account, it would be useful to discuss it also with his epistemic version of the information-theoretic approach in mind.

\section{Pitowsky's information-theoretic interpretation}

Pitowsky's approach is an epistemic interpretation of the quantum state, interpreted as a state of partial belief, in the sense of Bayesian probability. As we will see, differently from qBism, his view preserves at least part of the empirical character of the quantum state.

In Pitowsky's information-theoretic approach, closed subspaces of the Hilbert space correspond to possible states of the world, or events, while the quantum state represents an agent's uncertainty about it. In other words, here, as in qBism, the quantum state is a device for bookkeeping of probabilities (Pitowsky 2008, 4). Contrarily to Bub's information-theoretic approach, the quantum state is therefore a derived entity, and in fact a fundamental part of Pitowsky's interpretational work consists in the derivation of quantum probabilities from the structure of quantum events. The derivation starts from the axioms of QT as formulated by Birkoff and von Neumann (1936), of which the structure of quantum events is shown to be a model. According to the resulting representation theorem, the space of events $\mathrm{L}$ in quantum theory is the lattice of subspaces of a Hilbert space. The fact that, as a consequence of Gleason's theorem, quantum probabilities can be derivable from this sole structure, constitutes, according to Pitowsky, 'one of the strongest pieces of evidence in support of the claim that the Hilbert space formalism is just a new kind of probability theory' $(2006,14)$.

Finally, adopting Bayesian probability as a conceptual and formal background, means analysing probabilities through rational betting behaviour, i.e. they are measured 'by proposing a bet, and seeing what are the lowest odds he will accept' ((Ramsey 1926), as cited in (Pitowksy 2006, 15)).

Few clarifications are here in order, concerning the two dogmas and Pitowsky's stance about them.

In Pitowsky's view, the notion of measurement appears in the axioms of QT: QT does not make predictions about generic occurrences and correlations between them - in this betting game, agents can only bet on measurements results, and, between them, only those that can be considered facts, where not all measurement results are facts:

"by "fact" I mean here, and throughout, a recorded fact, an actual outcome of a measurement. Restricting the notion of "fact" in this way should not be understood, at this stage, as a metaphysical thesis about reality. It is simply the concept of "fact" that is analytically related to our notion of 
"event", in the sense that only a recordable event can potentially be the object of a gamble." (2006, 10)

If a measurement is performed of which no result is registered, then there is no fact of the matter about the result of that measurement. This does not mean that according to Pitowsky such results do not exist, but simply that they are not 'events' or 'facts' over which the theory is allowed to make predictions. So, yes, Pitowsky's interpretation violates the first dogma, but it is not completely clear what is the irreplaceable role of the notion of measurement, for which the latter can't be analysed in terms of more fundamental notions.

The second clarification concerns the second dogma, i.e. the reality of the quantum state. We have said that, in Pitowsky's view, the quantum state represents a bookkeeping device for keeping track of probabilities, which are different from classical probabilities. As we have seen, the risk for an epistemic view of the quantum state as a bookkeeping device is that it turns out being void of physical content, or, even worse, instrumentalist; part of Pitowsky's conceptual work is therefore devoted to stressing how this approach avoids it.

One way to distance from instrumentalism is by stressing the explanatory power of quantum Bayesianism. Under an instrumentalist view (e.g. in the textbook approach to QT), in fact, the question 'why do the quantum events do not conform to classical probabilities?' has no answer. The information-theoretic view, on the other hand, have more tools to provide such an explanation, i.e. it is realist towards the structure of quantum gambles, i.e. towards the structure of Hilbert space

"the Hilbert space, or more precisely, the lattice of its closed subspaces, [is] the structure that represents the "elements of reality" in quantum theory" (Pitowsky 2006, 4)

"Instrumentalists often take their "raw material" to be the set of space-time events: clicks in counters, traces in bubble chambers, dots on photographic plates, and so on. Quantum theory imposes on this set a definite structure. Certain blips in space-time are identified as instances of the same event. Some families of clicks in counters are assumed to have logical relations with other families, etc. What we call reality is not just the bare set of events, it is this set together with its structure, for all that is left without the structure is noise. [...]

It is one thing to say that the only role of quantum theory is to "predict experimental outcome" and that different measurements are "complementary." It is quite another thing to provide an understanding of what it means for two experiments to be incompatible, and yet for their possible outcomes to be related; to show how these relations imply the uncertainty principle; and even, finally, to realize that the structure of events dictates the numerical values of the probabilities (Gleason's theorem).” (Pitowksy 2003, 412) 
The fact that such a structure has a physical reality allows the above-cited explanations to be genuine physical explanations rather than collapsing to the kind of explanations given by logic or formal epistemology. Said physical explanations are not dynamical, but structural, in the sense of (Felline 2010): the explanandum phenomenon is explained as the instantiation of a physical structure, fundamental in the sense that it is not inferable from the properties and behaviour of underlying entities.

Pitowsky's realism, however, goes further than this. We have said before that, according to Pitowsky's account, only registered measurement results are considerable facts, about which the theory can make predictions. This claim, however, has an exception. Although probabilities are epistemic, in this view there are some 'hybrid' cases where the quantum state also describes an objective outside reality, and its predictions can be taken as genuine facts:

"In the Bayesian approach what constitutes a possible event is dictated by Nature, and the probability of the event represents the degree of belief we attach to its occurrence. This distinction, however, is not sharp; what is possible is also a matter of judgment in the sense that an event is judged impossible if it gets probability zero in all circumstances. In the present case we deal with physical events, and what is impossible is therefore dictated by the best available physical theory. Hence, probability considerations enter into the structure of the set of possible events. We represent by 0 the equivalence class of all events which our physical theory declares to be utterly impossible (never occur, and therefore always get probability zero) and by 1 what is certain (always occur, and therefore get probability one)." (Pitowsky 2006, 5).

So, according to this stipulations, not only registered results are facts, but also results that have probability 1 . In this case, the quantum state does actually represent something in the world.

This traces a crucial difference between Pitowsky's information-theoretic interpretation and Qbism. In the latter, given the strict Bayesian reading of probabilities, probability 1 only means that the person who associates this probability to an event $\mathrm{E}$ very strongly beliefs the occurrence of $\mathrm{E}$ (same thing for probability 0 ).

According to Pitowsky's analysis of the measurement problem, adopting an epistemic view is sufficient to explain away the measurement problem. In fact, the claim that the quantum state is a mental state blocks, at a first analysis, the consequences that we have seen in Bub's ontic approach.

This, however, is not a viable solution for Pitowsky's approach, due to the limited realism he acknowledges towards the quantum state.

In order to concretely see why, take again HH's argument and the predictions for the measurement of $\mathrm{O}$. In a partially objective reading of the state like Pitowsky epistemic view, the fact that Wigner associates probability 1 to the result YES means that such result is an objective fact 
about reality. But if this is so, then in Pitowsky's view there is a fact of the matter about what will be the result of the measurement of $\mathrm{O}$. But if this is so, Friend is objectively wrong.

But this, we know, would mean adopting a non-collapse view of QT.

\section{A Bohrian escape}

Recently (2018) Bub has formulated a new argument addressing the problem of incompatible predictions of QT in Wigner's Friend scenario. Bub does not directly cite HH's argument, and focuses instead on the more recent argument formulated in (Frauchiger and Renner 2018). In the following, for simplicity, I will keep using the argument as formulated by $\mathrm{HH}$.

Bub takes a stance about the notion of measurement that is very much in line with Pitowsky's view about genuine facts. Accordingly, QT does not apply to, nor make predictions about, generic events, but only about measurement results. Here, as in Pitowsky, the notion of measurement is substantially revised:

"A quantum "measurement" is a bit of a misnomer and not really the same sort of thing as a measurement of a physical quantity of a classical system. It involves putting a microsystem, like a photon, in a situation, say a beamsplitter or an analyzing filter, where the photon is forced to make an intrinsically random transition recorded as one of two macroscopically distinct alternatives in a device like a photon detector. The registration of the measurement outcome at the Boolean macrolevel is crucial, because it is only with respect to a suitable structure of alternative possibilities that it makes sense to talk about an event as definitely occurring or not occurring, and this structure is a Boolean algebra." (Bub 2018, 6)

So, on the one hand Bub provides a physical characterization that partially explicates the notion of measurement (it involves an intrinsically random transition for the measurement system, whose final state must be registered macroscopically); on the other hand, the first dogma is still violated, since there is no criterion for deciding whether or not a process counts as measurement (we don't know when a photon is forced to make an intrinsically random transition).

Bub explains the problems in Wigner's Friend scenario as originated by the structure of quantum events, composed by a "family of "interwined" Boolean algebras, one for each set of commuting observables $[\ldots]$. The interwinement precludes the possibility of embedding the whole collection into one inclusive Boolean algebra, so you can't assign truth values consistently to the propositions about observable values in all these Boolean algebras" (Bub 2018, 5)

So, the algebra of observables is non-Boolean, but each observable (each measurement) picks up a Boolean algebra which associates different probabilities to same observables. In our thought 
experiment: Friend's and Wigner's measurements pick up different Boolean algebras, that can't be consistently embedded into one single coherent framework.

According to Bub only one Boolean algebra provides the correct framework for the description of the state. In order to justify the selection of one single Boolean algebra, Bub starts from reminding how George Boole introduced Boolean constraints on probability as "conditions of possible experience". He therefore takes a 'Bohrian' perspective and suggests that the choice of a unique Boolean algebra is imposed by the necessity to "tell others what we have done and what we have learned" (Bohr, as cited in Bub 2018, 9)

The correct quantum state to associate to a system, therefore, is the one corresponding to what Bub calls the ultimate measurement (and relative ultimate observer). It is important to notice right from the start, and we will insist on this point later along the way, that in order for HH's objection to be met, all observers (not only the ultimate observer) must apply the same Boolean algebra, on pain of falling again into a scenario with inconsistent predictions.

But who is the ultimate observer in HH's thought experiment?

The only criterion for the identification of the ultimate measurement/observer is that the result of the measurement must be registered macroscopically.

"In a situation, as in the [Hagar and Hemmo] argument, where there are multiple candidate observers, there is a question as to whether [Friend is] "ultimate observer[..]," or whether only Wigner [is]". The difference has to do with whether [Friend] perform measurements of the observables $[\mathrm{P}]$ with definite outcomes at the Boolean macrolevel, or whether they are manipulated by Wigner [...] in unitary transformations that entangle [Friend] with systems in their laboratories, with no definite outcomes for the observables A and B. What actually happens to [Friend] is different in the two situations."

It is important to clarify, about this passage, that the exclusive character of the disjunction 'Friend performs measurements registered at the macrolevel or Wigner manipulates observables' is misleading. The two disjuncts are actually not mutually excluding: both can be true. In fact, let's assume, in HH's experiment, that both Wigner and Friend write down their result and are therefore ultimate observers. This is an unproblematic assumption, since, according to Bub's analysis, ${ }^{7}$ both Friend and Wigner act as ultimate observers, only in different moments of the experiment: in the

\footnotetext{
7 "If there are events at the macrolevel corresponding to definite measurement outcomes for Alice and Bob, then Alice and Bob represent "ultimate observers" and the final state of the combined quantum coin and qubit system is $|\mathrm{h}\rangle_{\mathrm{A}}|0\rangle_{\mathrm{B}}$ or $|t\rangle_{A}|0\rangle_{B}$ or $|t\rangle_{A}|1\rangle_{B}$, depending on the outcomes. If Wigner and Friend subsequently measure the super-observables X, Y on the whole composite Alice-Bob system (so they are "ultimate observers" in the subsequent scenario), the probability of obtaining the pair of outcomes $\{$ ok, ok $\}$ is $1 / 4$ for any of the product states $|\mathrm{h}\rangle_{\mathrm{A}}|0\rangle_{\mathrm{B}}$ or $|\mathrm{t}\rangle_{\mathrm{A}}|0\rangle_{\mathrm{B}}$ or $|\mathrm{t}\rangle_{\mathrm{A}}|1\rangle_{\mathrm{B}}$." (Bub 2018)
} 
first part, when Friend performs her measurement, she is the ultimate observer, while Wigner becomes the ultimate observer in the second part of the experiment.

Now, Wigner knows (as we know) that Friend will write down her measurements' results, and so that she is the legitimate ultimate observer in the first part of the experiment. He also knows that he has to take this assignment very seriously, given that "What actually happens to [Friend] is different" whether or not Friend is the ultimate observer and "the difference between the two cases $[\ldots]$ is an objective fact at the macrolevel" (Bub 2018, 12).

This means that Wigner knows that in the first part of the experiment a legitimate ultimate measurement is being performed in the lab, and with it the uncontrollable disturbance that characterize, in measurement processes, the passage from non-Booleanity to Booleanity. If we were to use Pitowsky's Bayesian framework, we could say that Friend's measurement is a gamble over which Wigner can bet. He does not clearly know what is the result of the experiment, but he knows that she definitely gets $\mathrm{a}+$ or a - result, because this is what the 'ultimate Boolean algebra' predicts.

The point I am trying to make is that, if QT is not a 'single user theory' (as it is not according to $\mathrm{Bub}$ ), and if the selection of a Boolean algebra does not apply uniquely to the ultimate observer (as it does not according to Bub), then after Friend's measurement, Both she and Wigner will have to predict that her measurement ends up either in the state (5) or in the state (6). The result of Friend's experiment is an objective fact about the world, which Wigner can't ignore.

The correct state that, according to this view, Wigner should associate to F+P after Friend's measurement is therefore a proper mixture of (5) and (6).

But if this is true, when Wigner makes his predictions about the measurement of $\mathrm{O}$, he should use the probabilities dictated by a proper mixture of (5) and (6), rather than those dictated by the entangled state (4).

But this, again, "would seem to require a suspension of unitary evolution in favour of an unexplained "collapse" of the quantum state" (3), which the information-theoretic view clearly rejects.

\section{Conclusions}

In this paper I have tested the solutions to the big measurement problem provided by different interpretations of QT as a theory about information, by analysing how they behave in a thought experiment à la Wigner's Friend.

As analytical tools for the examination of such performances, I've used the two claims that, according to Itamar Pitowsky, are at the basis of the conundrums of QT: the claim that the concept 
of measurement should not be used in the fundamental axioms of a physical theory and the claim that the quantum state represents a physical system, its properties and its dynamical evolution.

About the first dogma, $\mathrm{HH}$ argue that the conclusion of their argument is that black-box approaches are incoherent or not complete. If this is true, their argument can be seen as a justification of the first dogma, which, as a result, is not a dogma anymore: besides the already good reasons illustrated by John Bell, a good reason to adopt Bell's dictum is that black-box theories can't provide consistent predictions. In the previous sections I have acknowledged that qBism might have a way-out of HH's conclusions. Should we conclude that HH's argument fails in vindicating the first dogma?

I think this conclusion would be wrong. Bell's criticism of the notion of measurement is a reflection over physical theories: measurement can't appear in a fundamental physical theory because it is not a physically fundamental notion. It should be already clear that, since the conclusion of my analysis is that qBism is not a physical theory, the first dogma does not apply to it.

When seen, as it was intended, as a criterion for fundamental physics, Bell's dictum seems quite reasonable. However, it is hardly surprising that the notion of measurement, and measurement results, appear in the axioms of a theory about beliefs and about how to update beliefs with new experience. $^{8}$

The fact that Qbism is not affected by HH's argument, therefore, does not say much about the status of Bell's dictum as a dogma or as a justifiable request. As far as HH's argument is successful against physical black-box theories, it still provides a sufficient justification for the first dogma, which, according to $\mathrm{HH}$, is not dogmatic at all.

From the analysis put forward in this paper, therefore, Bell's dictum seems in great shape, and not a dogma at all.

Let's move to the analysis of the second dogma. The main target of this paper was the assumption, common to probabilistic and information-based approaches to QT, that the second dogma is responsible for the measurement problem, and that its rejection is sufficient to explain the problem away.

The failure of Bub's and Pitowsky's information-theoretic interpretations in the context of HH's thought experiment, on the one hand, and the discussed problems in the Qbist approach, show that this assumption is too simplistic: the claim that QT is about information does not solve the measurement problem, neither in the physical interpretation of the notion of information (as in Bub), nor in its epistemic interpretation (as in Pitowsky's).

\footnotetext{
${ }^{8}$ Although (Fuchs et al. 2014, p.2) for a reflection over the notion of measurement.
} 


\section{Acknowledgement}

The discussion of this paper at the Birmingham FraMEPhys Seminar series and at the MCMPWestern Ontario Workshop on Computation in Scientific Theory and Practice helped me to improve this paper. I am very thankful to the editors of this volume, and especially to Meir Hemmo for his valuable comments, and to Veronika Baumann for the same reason.

\section{Bibliography}

Bacciagaluppi, G.: A Critic Looks at QBism (2013). halshs-00996289

Baumann, V., Brukner, Č.: Wigner's friend as a rational agent (2019). arXiv preprint arXiv:1901.11274.

Bell, J. S.: Speakable and unspeakable in quantum mechanics. Cambridge University Press.

Birkhoff, G. von Neumann, J.: The logic of quantum mechanics. Ann. of Math. 37, 823 (1936)

Bub, J.: Bananaworld: Quantum mechanics for primates (2016). Oxford University Press.

Bub, J.: In defense of a "single-world" interpretation of quantum mechanics. Studies in History and Philosophy of Science Part B: Studies in History and Philosophy of Modern Physics. Online first (2018)

Bub, J., \& Pitowsky, I.: Two dogmas about quantum mechanics. Many worlds, 433-459. (2010)

Caves, C. M., Fuchs, C. A., Schack, R.: Unknown quantum states: the quantum de Finetti representation. J. of Math. Phys., 43(9), 4537-4559. (2002).

Caves, C. M., Fuchs, C. A., Schack, R.: Subjective probability and quantum certainty. Studies in History and Philosophy of Science Part B: Studies in History and Philosophy of Modern Physics, 38(2), 255-274. (2007).

Felline, L.: Quantum theory is not only about information. Studies in History and Philosophy of Science Part B: Studies in History and Philosophy of Modern Physics. (2018).

Frauchiger D., Renner R.: "Single-World interpretations of quantum mechanics cannot be selfconsistent," (2018) arXiv eprint quant- ph/1604.0742

Fuchs, C. A.: "QBism, the perimeter of quantum Bayesianism”. (2010). arXiv preprint arXiv:1003.5209.

Fuchs, C. A.: Notwithstanding Bohr, the Reasons for QBism. (2018) arXiv preprint 1705.03483.

Fuchs, C. A., Schack, R.: QBism and the Greeks: why a quantum state does not represent an element of physical reality. Physica Scripta, 90(1), 015104. (2014).

Fuchs, C. A., Mermin, N. D., Schack, R.: An introduction to QBism with an application to the locality of quantum mechanics. Am. J. of Phys., 82(8), 749-754. (2014). 
Fuchs, C. A., Stacey, B. C.: QBism: Quantum theory as a hero's handbook. In Proceedings of the International School of Physics “Enrico Fermi”, 197, 133-202). (2019, January).

Hagar, A.: Experimental metaphysics2: The double standard in the quantum-information approach to the foundations of quantum theory. Studies in History and Philosophy of Science Part B: Studies in History and Philosophy of Modern Physics, 38(4), 906-919. (2007).

Hagar, A., \& Hemmo, M.: Explaining the unobserved-Why quantum mechanics ain't only about information. Found. of Phys., 36(9), 1295-1324. (2006).

Maudlin, T.: Quantum Non-Locality and Relativity: Metaphysical Intimations of Modern Physics. (1997).

Myrvold, W.: "Philosophical Issues in Quantum Theory", The Stanford Encyclopedia of Philosophy (Fall 2018 Edition), Edward N. Zalta (ed.), URL = https://plato.stanford.edu/archives/fall2018/entries/qt-issues/ (2018)

Pitowsky, I.: Betting on the outcomes of measurements: a Bayesian theory of quantum probability. Studies in History and Philosophy of Science Part B: Studies in History and Philosophy of Modern Physics, 34(3), 395-414. (2003).

Pitowsky, I.: Macroscopic objects in quantum mechanics: A combinatorial approach. Phys. Rev. A, 70(2), 022103. (2004).

Pitowsky, I.: Quantum mechanics as a theory of probability. In Physical theory and its interpretation, 213-240. Springer, Dordrecht. (2006).

Ramsey, F.P.: Truth and Probability. (1926) reprinted In D. H. Mellor (ed.) F. P. Ramsey: Philosophical Papers. Cambridge: Cambridge University Press (1990)

Wallace, D.: What is orthodox quantum mechanics? (2016). arXiv preprint arXiv:1604.05973. 